\title{
Pseudomonas syringae Evades Host Immunity by Degrading Flagellin Monomers with Alkaline Protease AprA
}

\author{
Michiel J. C. Pel,, ${ }^{1,2}$ Anja J. H. van Dijken, ${ }^{1}$ Bart W. Bardoel, ${ }^{3}$ Michael F. Seidl, ${ }^{2,4}$ Sjoerd van der Ent, ${ }^{1,2}$ \\ Jos A. G. van Strijp, ${ }^{3}$ and Corné M. J. Pieterse ${ }^{1,2}$ \\ ${ }^{1}$ Plant-Microbe Interactions, Department of Biology, Faculty of Science, Utrecht University, Utrecht, The Netherlands; \\ ${ }^{2}$ Centre for BioSystems Genomics, Wageningen, The Netherlands; ${ }^{3}$ Medical Microbiology, University Medical Center \\ Utrecht, Utrecht, The Netherlands; ${ }^{4}$ Theoretical Biology and Bioinformatics, Department of Biology, Faculty of Science, \\ Utrecht University
}

Submitted 6 February 2014. Accepted 10 March 2014.

Bacterial flagellin molecules are strong inducers of innate immune responses in both mammals and plants. The opportunistic pathogen Pseudomonas aeruginosa secretes an alkaline protease called AprA that degrades flagellin monomers. Here, we show that AprA is widespread among a wide variety of bacterial species. In addition, we investigated the role of AprA in virulence of the bacterial plant pathogen $P$. syringae pv. tomato DC3000. The AprA-deficient DC3000 $\triangle$ aprA knockout mutant was significantly less virulent on both tomato and Arabidopsis thaliana. Moreover, infiltration of $A$. thaliana Col-0 leaves with DC3000 $\Delta$ aprA evoked a significantly higher level of expression of the defenserelated genes $F R K 1$ and $P R-1$ than did wild-type DC3000. In the flagellin receptor mutant $f l s 2$, pathogen virulence and defense-related gene activation did not differ between DC3000 and DC3000 $\triangle a p r A$. Together, these results suggest that AprA of DC3000 is important for evasion of recognition by the FLS2 receptor, allowing wild-type DC3000 to be more virulent on its host plant than AprA-deficient DC3000 $\triangle a p r A$. To provide further evidence for the role of DC3000 AprA in host immune evasion, we overexpressed the AprA inhibitory peptide AprI of DC3000 in A. thaliana to counteract the immune evasive capacity of DC3000 AprA. Ectopic expression of aprI in A. thaliana resulted in an enhanced level of resistance against wild-type DC3000, while the already elevated level of resistance against DC3000 $\triangle a p r A$ remained unchanged. Together, these results indicate that evasion of host immunity by the alkaline protease AprA is important for full virulence of strain DC3000 and likely acts by preventing flagellin monomers from being recognized by its cognate immune receptor.

For both plants and animals, distinguishing self from nonself is important for the activation of innate immune responses upon pathogen attack. In mammals and plants, microorganisms are recognized by pattern-recognition receptors (PRR)

Corresponding author: C. M. J. Pieterse; Telephone: +31 30253 6887; Fax: +31 30253 2837; E-mail: C.M.J.Pieterse@uu.nl

* The $\boldsymbol{e}$-Xtra logo stands for "electronic extra" and indicates that two supplementary figures and one supplementary table are published online.

(C) 2014 The American Phytopathological Society that detect common, highly conserved microbe-associated molecular patterns (MAMPs) such as lipopolysaccharides (LPS), peptidoglycans, chitin, elongation factor Tu (EF-Tu), and flagellin (Boller and Felix 2009; Pel and Pieterse 2013). Recognition of these conserved non-self molecules enables host cells to detect millions of microorganisms with a relatively small number of receptors and mediates a first line of defense against most of the nonadapted pathogens (Bardoel and Van Strijp 2011; Jones and Dangl 2006).

Flagellin is a highly conserved MAMP that can be recognized by both animal and plant cells. This protein forms the major part of the bacterial flagellum that enables bacterial motility. Each flagellum consists of thousands of flagellin molecules (Chevance and Hughes 2008; Gomez-Gomez and Boller 2002; Ramos et al. 2004; Samatey et al. 2001). Flagellin monomers can surround the bacteria due to spills during flagellum construction or due to damaging of the flagellar filaments (Gomez-Gomez and Boller 2002; Komoriya et al. 1999). It is these monomers that are recognized by mammalian and plant cells (Felix et al. 1999; Wyant et al. 1999). In mammals, the PRR Toll-like receptor 5 (TLR5) is required for detection of bacterial flagellin whereas, in plants, flagellin-sensitive 2 (FLS2) is responsible for flagellin recognition (Gomez-Gomez and Boller 2000; Hayashi et al. 2001). Both TLR5 and FLS2 recognize conserved parts of the flagellin molecule. However, the TLR5 recognition site was mapped to a cluster of 13 amino acids on both the $\mathrm{C}$ and $\mathrm{N}$ terminus of the flagellin monomers, while FLS2 recognizes a 22-amino-acid sequence in the $\mathrm{N}$ terminus of the protein (Felix et al. 1999; Smith et al. 2003). After binding of flagellin to TLR5 or FLS2, downstream signaling is triggered, leading to immune responses that are aimed at stopping the pathogen from entering the host tissue (Asai et al. 2002; Ronald and Beutler 2010).

For pathogen survival, suppression and evasion of host immune responses is of utmost importance. Many bacterial pathogens possess a type III secretion system that allows them to transfer proteins directly into host cells. These proteins are called effectors and generally contribute to virulence by suppressing host defense responses (Boller and He 2009). Besides suppression of host immunity, evasion of host immunity is an important virulence strategy as well. For example, the fungal plant pathogen Cladosporium fulvum secretes a LysM domaincontaining protein, called extracellular protein 6 (Ecp6). The LysM domains bind fungal chitin oligosaccharides, thereby preventing recognition of chitin by the host cell and avoiding 
activation of MAMP-triggered immune responses (Bolton et al. 2008; De Jonge et al. 2010).

The opportunistic pathogen Pseudomonas aeruginosa secretes an alkaline protease, designated AprA, which belongs to the serralysin family of the zinc metalloproteases (Miyoshi and Shinoda 2000). This protease is secreted by a type I secretion system and has been associated with virulence (Liehl et al. 2006; Parmely et al. 1990; Tommassen et al. 1992). Recently, we demonstrated that AprA actively degrades flagellin monomers (Bardoel et al. 2011). We hypothesized that AprA-mediated degradation of spilled flagellin monomers could be a bacterial strategy to evade host immunity. Here, we show that the bacterial alkaline protease AprA is widespread among many human- and plant-pathogenic bacteria, including the bacterial plant pathogen $P$. syringae pv. tomato DC3000. P. syringae pv. tomato is the causal agent of bacterial speck disease on a wide range of economically important crops and is rated as the number one bacterial pathogen based on scientific and economic importance (Mansfield et al. 2012). We provide evidence that the AprA protease of DC3000 plays an important role in the evasion of host immunity and is required for full virulence of DC3000 on both Arabidopsis thaliana and tomato (Solanum lycopersicum). Finally, we demonstrate that transgenic $A$. thaliana plants ectopically expressing the AprA inhibitor AprI of DC3000 show a significantly higher level of resistance to DC3000 infections, with which a novel tool is provided to protect plants against bacterial pathogens.

\section{RESULTS}

\section{AprA is widespread}

\section{among highly divergent pathogenic bacteria.}

The aprA gene encodes a 50-kDa zinc metalloprotease and, in $P$. aeruginosa, this gene is present in the same operon as the AprA inhibitor-encoding gene aprI and the type I secretion system encoding genes $\operatorname{aprD}$, aprE, and $\operatorname{aprF}$ (Stover et al. 2000). A similar operon has been described for the plant pathogen Dickeya dadantii (Duong et al. 1992; Guzzo et al. 1991), and the human pathogen Serratia marcescens has also been shown to possess aprA and aprI (Létoffé et al. 1989). Also, in the bacterial plant pathogen $P$. syringae pv. tomato DC3000, an operon containing aprA, aprI, aprD, aprE, and aprF can be identified (Fig. 1A) (Buell et al. 2003). The presence of an aprA homolog in these different $\gamma$-proteobacteria led to the hypothesis that this protease might be evolutionarily conserved.
To identify AprA homologs in other bacterial species, a protein BLAST followed by selection for serralysin-specific motifs was performed. This led to the identification of 134 AprA protein homologs from 102 different bacterial strains, of which many are pathogens on plants or mammals (Supplementary Table S1). Surprisingly, AprA is not limited to a specific group of bacteria but can be found in highly divergent bacterial species. Although most bacteria with an AprA homolog are $\alpha$-proteobacteria or $\gamma$-proteobacteria, AprA homologs of $\beta$-proteobacteria, cyanobacteria, and chlorobi also were identified.

For these AprA homologs to degrade flagellin monomers, as has been shown for AprA of P. aeruginosa (Bardoel et al. 2011), they need to be secreted. For P. aeruginosa and D. dadantii, it has been shown that this secretion depends on the type I secretion system that is encoded by $\operatorname{aprD}$, aprE, and $a p r F$, which are present in the same operon as aprA (Guzzo et al. 1991; Létoffé et al. 1990). To check whether the identified AprA homologs of the other 102 species are located in similar operons, the eight genes upstream and downstream of aprA were checked for the presence of protease inhibitors or genes involved in protein secretion. From the 53 bacterial species in the list of which the genomes have been sequenced and annotated, 28 had genes involved in protein secretion and 23 had genes encoding a protease inhibitor in the direct vicinity of aprA (Fig. 1B; Supplementary Fig. S1). We hypothesize that the aprA genes that are surrounded by both protease inhibitor and secretion system genes are likely to code for a protease with a function similar to that of AprA from P. aeruginosa.

\section{AprA of $P$. syringae pv. tomato $\mathrm{DC} 3000$ is able to cleave flagellin.}

Based on the operon structure of the aprA-containing operon, we postulated that DC3000 should be able to cleave flagellin molecules. To check this, the DC3000 aprA gene was fused to a His-tag and cloned into Escherichia coli, after which Histagged AprA protein was purified. Subsequently, $P$. aeruginosa flagellin monomers were treated in vitro for $30 \mathrm{~min}$ with purified His-tagged DC3000 AprA and assessed for integrity by Western blot analysis. Both N-terminal and C-terminal Histagged DC3000 AprA were able to cleave flagellin monomers in a way similar to $P$. aeruginosa AprA (Fig. 2) (Bardoel et al. 2011). To investigate whether DC3000 AprI was able to inhibit protease activity of DC3000 AprA, purified His-tagged DC3000 AprI was tested. Indeed, DC3000 AprI strongly inhibited the DC3000 AprA-mediated degradation of flagellin. Interestingly,

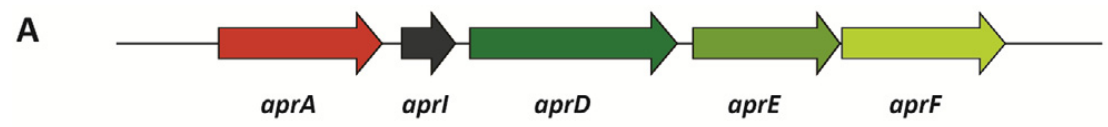

B

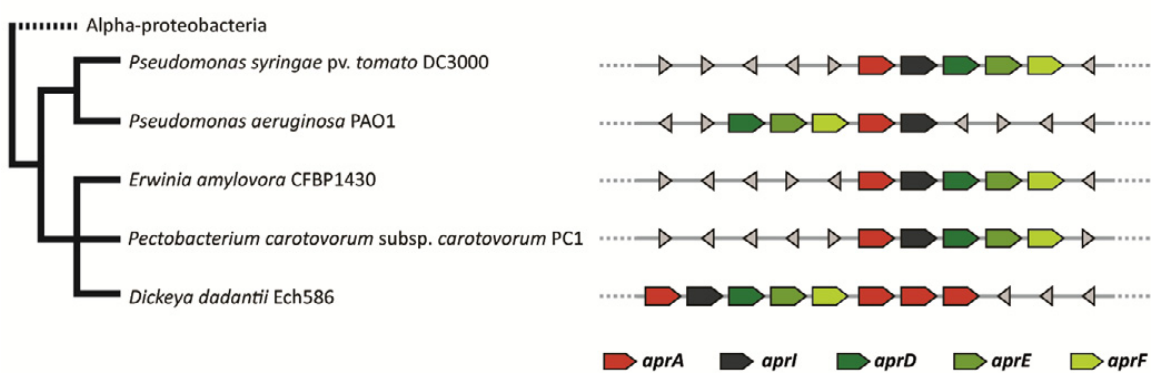

Fig. 1. AprA is present in highly divergent pathogenic bacteria. A, Schematic representation of the aprA operon in the genome of Pseudomonas syringae pv. tomato DC3000. In addition to aprA, the aprI gene encoding the AprA inhibitor AprI and the type I secretion system genes $\operatorname{aprD}$, aprE, and $a p r F$ are present. B, Genes surrounding the aprA homologs in the genomes of DC3000 (NP_793115), P. aeruginosa PAO1 (NP_249940), Erwinia amylovora CFBP1430 (YP_003532934), Pectobacterium carotovorum subsp. carotovorum PC1 (YP_003017132), and Dickeya dadantii Ech586 (YP_003333619). Genes that encode AprA homologs, AprI-like protease inhibitors, or type I secretion proteins are depicted in different colors. All other genes are shown in gray. The phylogenetic tree represents species taxonomy. 
purified AprI from $P$. aeruginosa did not inhibit DC3000 AprA activity, confirming previous observations that AprI activity is highly specific (Bardoel et al. 2012). The fact that both AprA from $P$. aeruginosa and AprA from DC3000 are able to cleave flagellin monomers suggests that this is a common feature for AprA homologs and may represent a conserved function of this protein in host immune evasion by bacterial pathogens.

\section{AprA is an important virulence factor}

\section{for $P$. syringae pv. tomato DC3000 on tomato.}

Because bacterial flagellin monomers are a substrate for DC3000 AprA, we hypothesized that AprA may function in the evasion of pathogen detection by the host plant. $P$. syringae pv. tomato DC 3000 is the causal agent of bacterial speck disease on tomato (Preston 2000). To test the role of DC3000 AprA in pathogen virulence, a DC3000 $\Delta a p r A$ knockout mutant was constructed by exchanging the wild-type aprA gene with a dysfunctional $\triangle a p r A$ gene in which a carbenicillin (carb) resistance cassette was inserted by homologous recombination (Supplementary Fig. S2A). In addition, the DC3000 $\triangle a p r A$ mutant was complemented with the AprA coding region under control of the constitutive nptII promoter (designated $\triangle a p r A+\mathrm{pN}$ ). The genotypes were confirmed using polymerase chain reaction (PCR). To test for AprA protease production, secretion, and activity, the different DC3000 strains were grown on tryptic soy agar containing milk powder. In this protease activity assay, wild-type DC3000 produced a halo and the formation of this halo could be blocked by the addition of DC3000 AprI, indicating that this halo is AprA dependent. DC3000 $\triangle$ aprA did not produce a halo in this protease activity assay, indicating that it does not produce active AprA. Halo production was restored to wild-type levels in the complementation DC3000 strain $\triangle a p r A+\mathrm{pN}$. This also demonstrates that the production of the type I secretion system proteins AprD, AprE, and AprF, of which the corresponding coding genes are located downstream of aprA in the same operon, was not affected in the DC3000 $\triangle a p r A$ knockout mutant.

To test the role of DC3000 AprA during bacterial infection of tomato, leaflets of 5-week-old tomato plants ('Moneymaker') were inoculated with DC3000 or DC3000 saprA and symptom development and bacterial growth was monitored. Symptoms on two opposing tomato leaflets 7 days after inoculation with DC3000 (left leaflet) or DC3000 $\Delta a p r A$ (right leaflet) are shown in Figure 3A. Inoculation with DC3000 $\Delta a p r A$ resulted in the development of less-severe symptoms, which was indicated by a reduced amount of cell death and chlorosis compared with that observed in leaflets that were inoculated with DC3000. In addition, growth of DC3000 $\Delta a p r A$ was significantly reduced compared with that of wild-type DC3000 (Fig. 3B). At 4 days after infection, a fourfold difference in bacterial growth could be observed, while this difference increased to almost 10-fold on day 7 after inoculation (Fig. 3B). These results indicate that the AprA protease is an important virulence factor for DC3000 during pathogenesis on tomato.

\section{Inactivation of flagellin by AprA is important for bacterial virulence on $A$. thaliana.}

Flagellin is a ubiquitous MAMP of bacterial pathogens that is recognized by the FLS2 receptor to confer basal immunity in plants (Ronald and Beutler 2010; Zipfel et al. 2004). In A. thaliana, FLS2-mediated closure of stomata is an early immune response that is effective when DC3000 enters the leaves via these natural openings, such as when plants are dip inoculated (Zeng and He 2010; Zipfel et al. 2004). Previously, it was shown that, upon pressure infiltration, growth of DC3000 in mutant $f l s 2$ plants does not differ from that in wild-type plants (Zipfel et al. 2004), suggesting that flagellin recognition does not contribute to the activation of post-invasion immune responses triggered by wild-type DC3000. We hypothesized that this is caused by DC3000 AprA activity that degrades elicitor-active flagellin, resulting in a redundant role for FLS2 in the inhibition of DC3000 growth once the pathogen is in the intercellular spaces. We reasoned that, if DC3000 $\triangle a p r A$ lacks the flagellin-degrading activity of AprA, it should evoke enhanced FLS2-dependent immune responses, resulting in inhibition of pathogen growth in pathogen-infiltrated leaves. To test this hypothesis, we pressure infiltrated Col-0 and fls2 with wild-type DC3000 and mutant DC3000 $\Delta a p r A$. As expected, no significant differences in growth of wild-type DC3000 could be observed in Col-0 and fls 2 plants (Fig. 3C), confirming previous findings (Zipfel et al. 2004). Interestingly, infiltration of Col-0 plants with DC3000 $\triangle a p r A$ resulted in a significantly lower bacterial growth than in plants infiltrated with the wild-type DC3000 strain (Fig. 3C), possibly because the AprA-deficient DC3000 $\Delta a p r A$ strain evoked a stronger defense response than wild-type DC3000. In the flagellin receptor mutant $f l s 2$, no significant difference could be observed between growth of DC3000 andvDC3000 $\Delta a p r A$. These results suggest that the reduced growth of DC3000 $\triangle a p r A$ in Col-0 is at least partly mediated through flagellin recognition by the plant and, thus, that AprA in wild-type DC3000 is involved in evasion of recognition by the FLS2 receptor when colonizing the intercellular spaces. Although no significant differences could be observed between $f l s 2$ plants inoculated with DC3000 and DC3000 $\triangle a p r A$, a small difference in growth between both $P$. syringae pv. tomato strains was consistently observed. Additionally, the difference in growth of DC3000 $\Delta$ aprA in Col- 0 versus $f l s 2$ was relatively minor. This might be caused by the fact that DC3000 produces multiple MAMPs (e.g., EFTu) (Kunze et al. 2004), which may also be sensitive to degradation by AprA. The complemented strain $P$. syringae pv. tomato DC3000 $\Delta a p r A+\mathrm{pN}$, which produced similar amounts of AprA in the protease activity plate assay, displayed a similar level of virulence as wild-type DC3000 (Fig. 3D), indicating that AprA function in this strain was restored. Together, these results suggest that AprA is important for full virulence of DC3000 on A. thaliana.

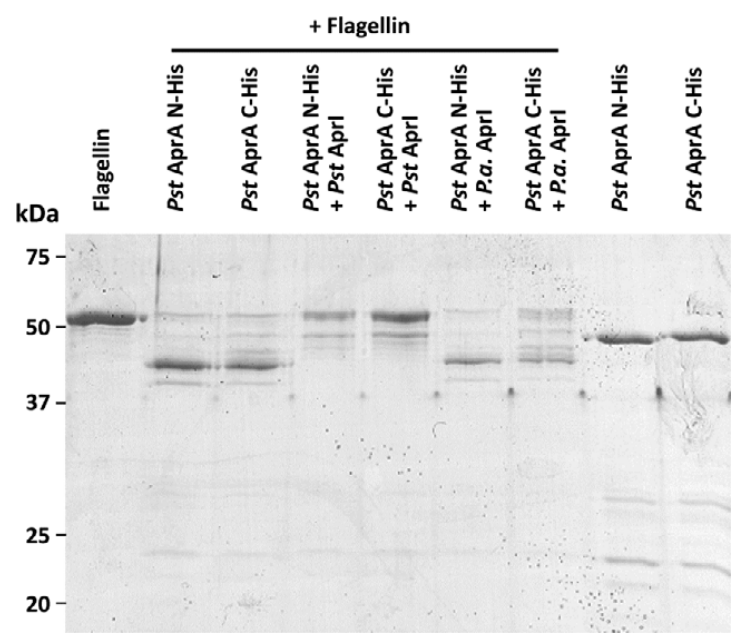

Fig. 2. Protease activity of Pseudomonas syringae pv. tomato DC3000 (Pst) AprA on bacterial flagellin. Flagellin at $250 \mu \mathrm{g} \mathrm{ml}^{-1}$ was treated for 30 min with DC3000 AprA at $10 \mu \mathrm{g} \mathrm{ml}^{-1}$ fused to an $\mathrm{N}$ - or C-terminal His-tag. The AprA inhibitor AprI from DC3000 or P. aeruginosa $(\mathrm{Pa})$ was added $\left(10 \mu \mathrm{g} \mathrm{ml}^{-1}\right)$ simultaneously with AprA when indicated. AprA protease activity on flagellin was analyzed by sodium dodecyl sulfate polyacrylamide gel electrophoresis and Coomassie staining. Untreated monomeric $P$. aeruginosa flagellin $\left(250 \mu \mathrm{g} \mathrm{m}^{-1}\right)$ runs at $52 \mathrm{kDa}$ in lane 1 . In lanes 8 and 9, purified DC3000 AprA N-His $\left(250 \mu \mathrm{g} \mathrm{ml}^{-1}\right)$ and DC3000 AprA C-His $\left(250 \mu \mathrm{g} \mathrm{ml}^{-1}\right)$ are loaded as controls. 
AprA delays the activation of plant defense responses.

When bacteria effectively degrade their own spill of flagellin monomers by producing AprA, they can escape detection by the host immune system. Following this rationale, we hypothesized that DC3000 $\triangle a p r A$ would be better recognized by $A$. thaliana than wild-type DC3000, and that this difference would at least partly be mediated by FLS2. To test this, Col-0 was pressure-infiltrated with wild-type DC3000 or mutant DC3000 $\triangle a p r A$, after which we monitored the expression of the flagellin-responsive defense-related genes FRK1 and $P R-1$ in both wild-type Col-0 and mutant $f l s 2$ plants. The MAMPresponsive gene $F R K 1$ was activated in Col-0 plants by wildtype DC3000 (Fig. 4A). However, FRK1 was activated to a significantly higher level upon inoculation by mutant DC3000 $\Delta a p r A$. When fls 2 mutant plants were inoculated with these bacterial strains, this difference in $F R K 1$ gene activation was not observed. In Col-0, the defense-related gene $P R-1$ was also activated to a significantly higher level by DC3000 $\Delta a p r A$ compared with DC3000, whereas this difference was not observed in mutant fls2 (Fig. 4B). These results corroborate the findings shown in Figure 3 and point to a role for DC3000 AprA in reducing the activation of host defenses that are mediated via the flagellin receptor FLS2.

Ectopic expression of the bacterial AprA inhibitor AprI in $A$. thaliana confers enhanced disease resistance against $P$. syringae pv. tomato $\mathrm{DC} 3000$.

To further validate the importance of AprA for full virulence of DC3000, we constructed transgenic A. thaliana lines over- expressing the bacterial AprA inhibitor AprI. Purified DC3000 AprI is able to inactivate DC3000 AprA activity in vitro and in vivo (Fig. 2). Therefore, we hypothesized that overexpression of DC3000 AprI in planta would inactivate DC3000 AprA and subsequently lead to a stronger recognition by the host and, thus, enhanced disease resistance. To test this, we constructed transgenic A. thaliana plants in which DC3000 AprI was overexpressed and targeted to the plant apoplast. Using the constitutively expressed Cauliflower mosaic virus $35 \mathrm{~S}$ promoter, two different constructs were made, one encoding AprI with its native bacterial signal peptide (SP) (designated 35Sp:aprI [SP AprI]) and one with the SP of PR-1 from A. thaliana (designated 35Sp:aprI [SP PR-1]). Wild-type A. thaliana and AprIexpressing A. thaliana lines were inoculated with DC3000 and DC3000 $\triangle a p r A$. DC3000 $\Delta a p r A$ performed significantly less well on Col-0 than DC3000 (Fig. 5), confirming the findings presented in Figure 3. Interestingly, the growth rate of DC3000 on the AprI-overexpressing lines was significantly reduced and reached a level similar to that of DC3000 $\Delta a p r A$. In the AprIoverexpressing plants, growth of DC3000 $\Delta a p r A$ was similar to that in Col-0 and no difference in growth could be observed between DC3000 and DC3000 saprA, suggesting that plantproduced AprI does not directly act on bacterial growth but via an effect on bacterially produced AprA. These results show that ectopic expression of bacterial aprI in A. thaliana affects virulence of AprA-producing DC3000 but not that of mutant DC3000 $\triangle a p r A$. This further strengthens our notion that AprA is an important virulence factor involved in evasion of host immunity. These results also indicate that production of the
A
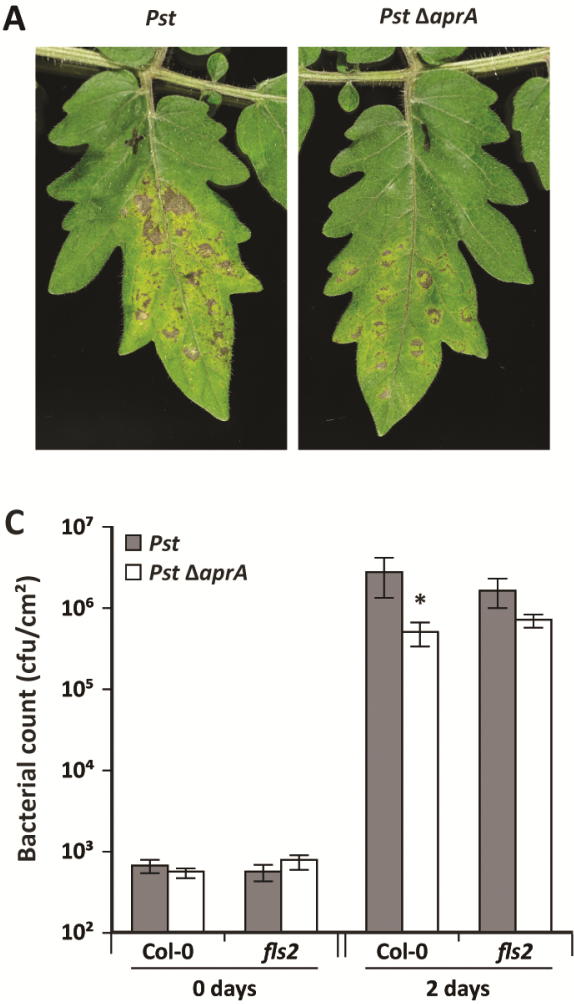

B
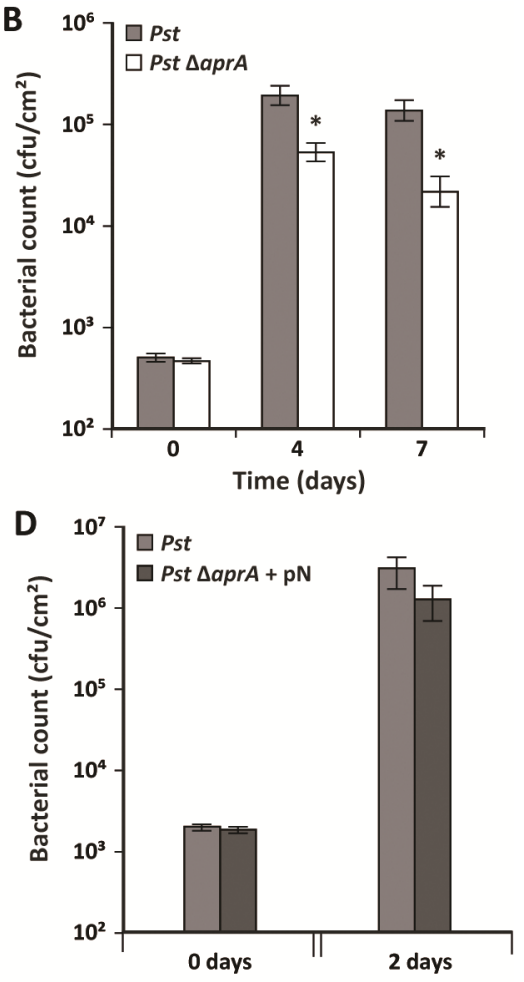

Fig. 3. AprA is important for full virulence of Pseudomonas syringae pv. tomato DC3000. A, Bacterial speck disease symptoms on tomato caused by DC3000 and DC3000 $\triangle a p r A, 7$ days postinoculation. Five-week-old 'Moneymaker' tomato plants were pressure infiltrated with DC3000 or DC3000 $\triangle a p r A$ in two opposing leaflets of the same tomato leaf. B, Growth of DC3000 and DC3000 $\triangle a p r A$ in tomato leaves. The number of bacteria in the intercellular space of pressure-infiltrated tomato leaves was determined directly after and 4 and 7 days after inoculation. Asterisks indicate statistically significant differences (Student's $t$ test, $P<0.05$ ). C, Effect of AprA on bacterial proliferation in Arabidopsis thaliana wild-type Col-0 and the flagellin receptor mutant fls2. Leaves of Col-0 and fls 2 were pressure-infiltrated with DC3000 or DC3000 $\Delta a p r A$. Bacterial counts were determined directly after inoculation and two days later. An asterisk indicates a statistically significant difference between DC3000 and DC3000 $\Delta a p r A$ (Kruskal-Wallis test followed by pairwise comparisons; $P<0.05)$. D, Complementation of DC3000 $\triangle$ aprA. Col-0 plants were inoculated with DC3000 or DC3000 $\Delta a p r A$ mutant bacteria complemented with aprA under control of the constitutive nptII promoter $(\mathrm{pN})$. Bacterial proliferation was determined 2 days after inoculation. No significant differences could be observed (analysis of variance followed by Fisher's least significant difference test; $P<0.05$ ). Experiments were repeated with similar results. 
AprA inhibitor AprI by plants is an effective strategy to suppress bacterial infections.

\section{DISCUSSION}

Many bacteria produce and secrete proteases. The proteases produced by pathogenic bacteria are often associated with bacterial virulence, especially the zinc metalloproteases (Miyoshi and Shinoda 2000). A famous example of this is the zinc metalloprotease anthrax toxin lethal factor produced by Bacillus anthracis, of which the protease catalytic site is required for its lethal activity (Klimpel et al. 1994). Also, $P$. aeruginosa secretes several proteases that are involved in bacterial virulence, one of which is AprA. AprA has been shown to degrade the human cytokines interferon $\gamma$ and tumor necrosis factor $\alpha$ that are important for host immune activation (Parmely et al. 1990). Furthermore, AprA of $P$. aeruginosa has been shown to degrade monomeric flagellin molecules that are recognized by the immune receptors TLR5 and FLS2 in human and plant cells, respectively (Bardoel et al. 2011). During growth in their hosts, flagellin monomers can surround the bacteria due to spills during flagellum construction or due to damaging of the flagellar filaments (GomezGomez and Boller 2002; Komoriya et al. 1999). Hence, degradation of flagellin by bacterial AprA would be an effective mechanism of bacterial pathogens to evade flagellin-mediated recognition by the host immune system. Here, we demonstrated that AprA is, indeed, important for full virulence of the bacterial plant pathogen $P$. syringae pv. tomato DC3000. The $\Delta a p r A$ mutant of DC3000 showed reduced growth in tomato and A. thaliana (Fig. 3) and triggered a significantly stronger ex-
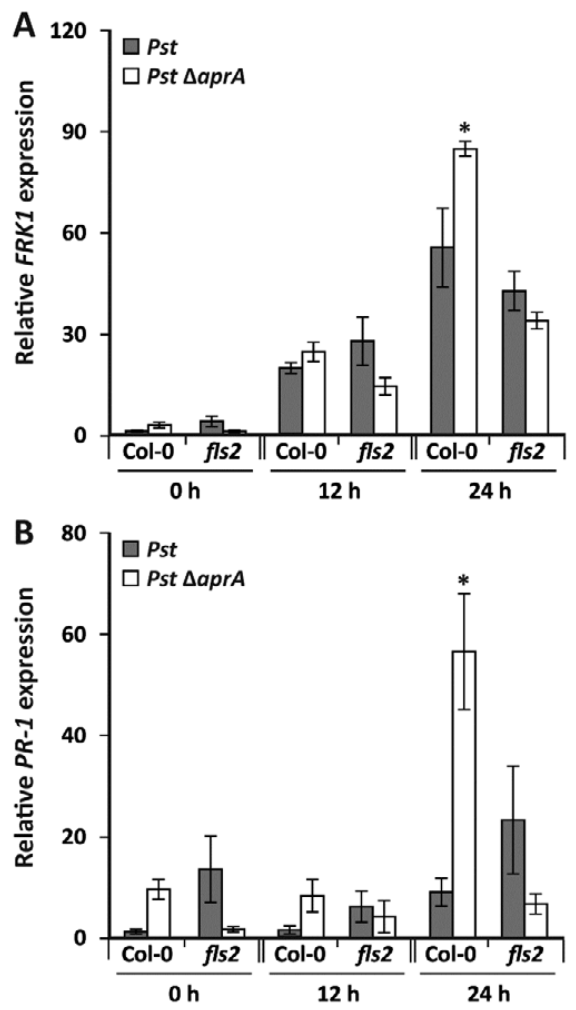

Fig. 4. Role of DC3000 AprA in evasion of defense-related gene activation in Arabidopsis thaliana. Quantitative reverse-transcriptase polymerase chain reaction analysis of the defense-related genes $\mathbf{A}$, FRK1 and B, PR-1 in 5-week-old Col-0 and fls2 plants that were inoculated with DC3000 or DC3000 $\Delta a p r A$. Leaf tissue was harvested for RNA analysis at 0 and $24 \mathrm{~h}$ after inoculation. Gene expression levels are relative to the expression at $t=0$ in Col-0. Asterisks indicate statistically significant differences (analysis of variance followed by Fisher's least significant difference test; $P<$ 0.05). Experiments were repeated with similar results. pression of the defense-related genes $F R K 1$ and $P R-1$ compared with wild-type DC3000 bacteria (Fig. 4). The fact that this reduction in pathogen growth and enhanced defense-related gene expression was not observed in the flagellin receptor mutant fls 2 suggests that flagellin is an important target of AprA in this immune evasive process.

When A. thaliana wild-type or fls 2 mutant plants were inoculated with wild-type DC3000 using pressure infiltration, no differences in bacterial growth were found (Fig. 3C), confirming previous findings (Zipfel et al. 2004). We hypothesized that this may be caused by inactivation of flagellin by DC3000 AprA in the intercellular space, rendering the role of FLS2 in pathogen detection in this stage of the infection to be redundant. When DC3000 $\Delta a p r A$ was pressure infiltrated in Col-0, the pathogen grew significantly less well compared with wild-type DC3000; whereas, in mutant $f l s 2$, no significant difference in growth between DC3000 and DC3000 $\Delta a p r A$ could be observed (Fig. $3 C)$. This suggests that DC3000 $\triangle a p r A$ is detected in the intercellular space in an FLS2-dependent manner, while wild-type DC3000 is not, pointing to a role for AprA in the evasion of FLS2-mediated pathogen detection. Despite the fact that growth of DC3000 and DC3000 $\triangle a p r A$ did not differ significantly on mutant fls 2 plants, DC3000 $\Delta a p r A$ consistently showed a small reduction in growth on fls 2 compared with wild-type DC3000. Also, the difference in growth of DC3000 $\triangle a p r A$ on Col-0 versus $f l s 2$ was relatively minor (Fig. 3C). Because DC3000 produces multiple proteinaceous MAMPs (e.g., EF-Tu) (Kunze et al. 2004), we hypothesize that degradation of one or more of these MAMPs by AprA may also contribute to the observed AprA-mediated effects on DC3000 virulence.

Escaping recognition is important for microbes with a pathogenic lifestyle and, because MAMPs such as bacterial flagellin are recognized by host PRR, they are under selective pressure (Pel and Pieterse 2013). However, changes in the conserved part of flagellin that is recognized by the FLS2 receptor result in loss of bacterial mobility (Smith et al. 2003); hence, it is not illogical that bacteria evolved other means by which they can escape from flagellin-mediated recognition by the host. AprA is able to degrade spilled flagellin molecules, which prevents recognition by the host and leads to enhanced bacterial virulence without affecting the bacterial mobility (Bardoel et al.

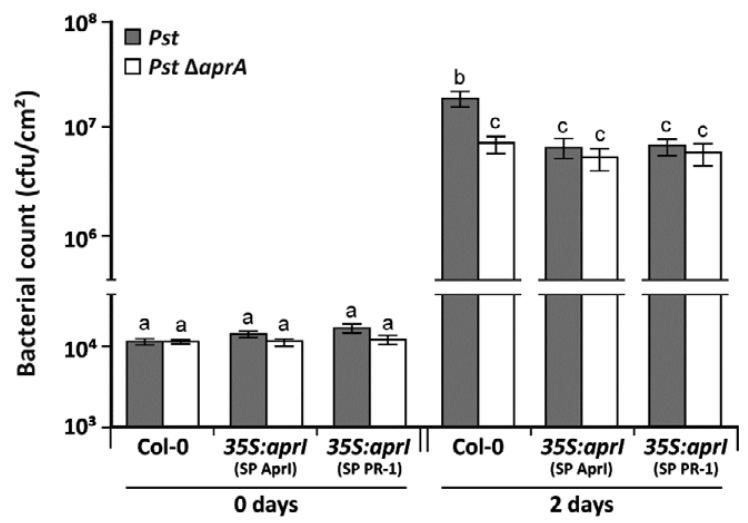

Fig. 5. Effect of ectopic expression of AprA inhibitor AprI in Arabidopsis thaliana on disease resistance. Data represent growth of Pseudomonas syringae pv. tomato DC3000 in leaves of transgenic 35Sp:aprI (SP AprI) and 35Sp:aprI (SP PR-1) A. thaliana plants that overexpress the aprI gene that encodes the AprA inhibitor AprI of DC3000 with its own signal peptide (SP AprI) or with the signal peptide of PR-1 (SP PR-1). Leaves of Col-0- and aprI-overexpressing plants were inoculated with DC3000 or DC3000 saprA. Bacterial counts were determined directly after pressure infiltration and 2 days later. Different letters indicate statistically significant differences (Kruskal-Wallis test followed by pairwise comparisons; $P<$ 0.05). Experiments were repeated with similar results. 
2011). Therefore, this active immune evasive strategy may be widespread among bacteria. This is supported by the fact that bacterial pathogens from many different taxonomic groups possess homologous aprA genes (Fig. 1). In addition to bacterial pathogens, many nonpathogenic bacteria also were shown to possess homologs of aprA (e.g., the biological control strain $P$. fluorescens SBW25 and the nitrogen-fixing Ensifer meliloti 1021 that live in close contact with host organisms) (Galibert et al. 2001; Silby et al. 2009). This suggests that AprA-mediated immune evasion might also play a role in the interaction between plants and beneficial bacteria.

The AprA inhibitor gene aprI is encoded by the same operon as aprA in the majority of the bacterial species in which an aprA homolog was identified (Fig. 1B). Based on its SP, AprI is predicted to be transported to the periplasmic space and, for $D$. $d a$ dantii, this cellular localization has been confirmed (Létoffé et al. 1990). However, AprA is transported over the two bacterial membranes at once by the type I secretion system. Therefore, AprI is theoretically never in contact with AprA and, thus, cannot bind this protease to inhibit its function. Hence, the function of AprI for the bacterium remains unclear. Because AprA is important for full virulence of DC3000 on both A. thaliana and tomato and possibly for many plant-pathogen interactions with AprA producing bacterial pathogens, we hypothesized that AprI could serve as an important tool to enhance the level of resistance in plants to these bacterial pathogens. Indeed, production of AprI by transgenic A. thaliana plants reduced growth of DC3000 in the intercellular space but not that of DC3000 DaprA (Fig. 5), confirming our notion that AprA is important in preventing pathogen detection by the plant immune system.

Inactivating bacterial AprA by AprI appears to be a good strategy to enhance the level of disease resistance against AprA-producing pathogens. Whether AprI can be successfully used against different bacterial infections depends largely on the specificity of this protease inhibitor. It has been shown that AprI of $D$. dadantii can not only inhibit the three proteases produced by itself but also inhibit AprA of S. marcescens, which is an opportunistic pathogen on humans (Létoffé et al. 1989). However, AprI of DC3000 cannot inhibit AprA of $P$. aeruginosa and vice versa (Fig. 2) but the exchange of only a few residues of AprI from DC3000 and P. aeruginosa can abolish the observed specificity (Bardoel et al. 2012). Hence, developing AprI-based strategies may provide novel tools to protect plant and mammalian hosts against infection by AprAproducing bacterial pathogens.

\section{MATERIALS AND METHODS}

\section{Bacterial strains and plasmids used.}

The following bacterial strains and plasmids were used: $P$. syringae pv. tomato DC3000 (50 $\mu \mathrm{g}$ of rifampicin per milliliter [rif ${ }^{50}$ ) (Whalen et al. 1991), DC3000 $\Delta a p r A$ (rif ${ }^{50}, 100 \mu \mathrm{g}$ of carbenicillin per milliliter $\left.\left[\mathrm{carb}^{100}\right]\right)$, DC3000 $\Delta a p r A+$ pBBR1MCS-5_aprAp:aprA (rif ${ }^{50}$, carb $^{100}, 50 \mu \mathrm{g}$ of gentamicin per milliliter $\left.\left[\mathrm{gen}^{50}\right]\right)$, DC3000 $\Delta$ aprA + pBBR1MCS5_nptIIp:aprA $\left(\mathrm{rif}^{50}\right.$, carb $^{100}$, gen $\left.^{50}\right)$, Escherichia coli Rosetta gami (DE3) pLysS (30 $\mu \mathrm{g}$ of chloramphenicol per milliliter, 50 $\mu \mathrm{g}$ of streptomycin per milliliter $\left[\mathrm{str}^{50}\right], 10 \mu \mathrm{g}$ of tetracycline per milliliter) (Novagen, Darmstadt, Germany), E. coli DH5 $\alpha$ (Bethesda Research Laboratories 1986), E. coli S17-1 $\lambda$ pir (Herrero et al. 1990), E. coli HB101 pRK2073 (50 $\mu \mathrm{g}$ of spectinomycin per milliliter) (Boyer and Roulland-Dussoix 1969; Figurski and Helsinki 1979), Agrobacterium tumefaciens EHA105 pJIC.SaRep, pUC4K (100 $\mu \mathrm{g}$ of ampicillin per milliliter, $\left[\mathrm{kan}^{25}\right]$ ) (Vieira and Messing 1982), pCR-BluntII-TOPO ( $\mathrm{kan}^{25}$; Life Technologies, Carlsbad, CA, U.S.A.), pKNG101 $\left(\operatorname{str}^{50}\right)$ (Kaniga et al. 1991) and pBBR1MCS-5 (gen $\left.{ }^{50}\right)$ (Kovach et al. 1994), pFAST-R02 and pFAST-R05 (Shimada et al. 2010), and pENTRD-TOPO (Life Technologies).

\section{Identification of AprA homologs.}

To identify homologs of DC3000 AprA (National Center for Biotechnology Information NP_249940), a pBLAST (Altschul et al. 1990) with AprA from D $100 \mu \mathrm{g} / \mathrm{ml}$ ampicillinC3000 against the RefSeq database was performed. The best thousand hits were checked for the presence of the motifs HEXXHXXG XXH and SXMSY (Miyoshi and Shinoda 2000). For the available genomes the eight genes up- and downstream of the AprA homolog were checked, using MGV2 (Kerkhoven et al. 2004) or manually, for the presence of serralysin-like proteins, protease inhibitors, and genes encoding proteins involved in protein secretion.

\section{AprA and AprI protein purification and flagellin degradation.}

The aprA and aprI genes from DC3000 were cloned into the plasmid pET302 and pRSETB, respectively, and transformed to E. coli Rosetta gami (DE3) pLysS (Novagen), as described (Bardoel et al. 2012). The recombinant proteins were purified using a His-trap column under denaturing (AprA) or native (AprI) conditions. AprA was renatured, as described previously (Bardoel et al. 2011), and proteins were dialyzed against phosphate-buffered saline (PBS). AprI from $P$. aeruginosa was produced as described (Bardoel et al. 2011). Recombinant flagellin from $P$. aeruginosa was purified as described (Bardoel et al. 2011). Flagellin $\left(250 \mu \mathrm{g} \mathrm{ml}^{-1}\right)$ was treated with DC3000 AprA at $10 \mu \mathrm{g} \mathrm{ml}^{-1}$ in PBS for $30 \mathrm{~min}$ at $37^{\circ} \mathrm{C}$ with or without AprI at $10 \mu \mathrm{g} \mathrm{ml}^{-1}$.

\section{Construction of the $P$. syringae pv. tomato DC3000 $\triangle a p r A$ knockout mutant.}

The aprA gene was isolated and cloned into pCR-BluntIITOPO (Life Technologies). An amp/carb resistance cassette was isolated from pUC4K (Taylor and Rose 1988) and was then cloned into the aprA gene in pCR-BluntII-TOPO. The aprA gene with the amp/carb resistance cassette inserted was then cloned from pCR-BluntII-TOPO to pKNG101 (Kaniga et al. 1991) and the resulting plasmid was introduced into $E$. coli S17-1 $\lambda$ pir. Next, the pKNG101 plasmid was introduced into DC3000 using triparental mating to create DC3000 saprA. This was confirmed by PCR. For complementation of aprA in DC3000 $\triangle a p r A$, the coding sequence of aprA was cloned by PCR (Phusion, Bioké, Leiden, The Netherlands) from DC3000 genomic DNA. The resulting product was cloned into the broad-host-range vector pBBR1MCS-5. The nptIIp:aprA complementation construct was created by insertion of the nptII promoter fragment, obtained from pUC4KIXX. DC3000 $\Delta a p r A$ was transformed with these plasmids by triparental mating.

\section{Assessment of protease activity.}

Overnight cultures of wild-type DC3000 and mutant DC3000 $\triangle a p r A$ (in King's B $[\mathrm{KB}]$ medium at $28^{\circ} \mathrm{C}$ ) were centrifuged at 4,000 rpm and resuspended in $10 \mathrm{mM} \mathrm{MgSO}_{4}$ to an optical density at $600 \mathrm{~nm}\left(\mathrm{OD}_{600}\right)=0.01\left(1 \times 10^{7} \mathrm{CFU} \mathrm{ml}{ }^{-1}\right)$. Of each strain, a $1-\mu 1$ droplet of the bacterial suspension was placed on tryptic soy agar containing skimmed milk powder (Elk, Campina, Amersfoort, The Netherlands) at $0.01 \mathrm{~g} \mathrm{ml}^{-1}$. Where indicated, a $50-\mu \mathrm{l}$ droplet of a $20-\mu \mathrm{g} \mathrm{ml}^{-1}$ AprI solution was placed preceding bacterial inoculation. Subsequently, the bacteria were grown at $16^{\circ} \mathrm{C}$ for 4 days.

\section{Cultivation of plants.}

Arabidopsis thaliana seed (Col-0 or fls2 [Col-0] SALK 141277; Shan et al. 2008) were sown on quartz sand. Two- 
week-old seedlings were transferred to $60-\mathrm{ml}$ pots containing a sand-potting soil mixture that had been autoclaved twice for 20 min with a 24-h interval (Van Wees et al. 2013). Plants were cultivated in a growth chamber with a cycle of 10-h days $\left(100 \mu \mathrm{E} \mathrm{m}^{-2} \mathrm{~s}^{-1}\right.$ at $\left.21^{\circ} \mathrm{C}\right)$ and $14-\mathrm{h}$ nights $\left(20^{\circ} \mathrm{C}\right)$ at $70 \%$ relative air humidity $(\mathrm{RH})$. For the tomato experiments, Solanum lycopersicum 'Moneymaker' was used. Seed were sown in $500-\mathrm{ml}$ pots containing potting soil and placed in a growth chamber with a cycle of $16-\mathrm{h}$ days $\left(200 \mu \mathrm{E} \mathrm{m} \mathrm{m}^{-2} \mathrm{~s}^{-1}\right.$ at $\left.21^{\circ} \mathrm{C}\right)$ and 8 -h nights $\left(20^{\circ} \mathrm{C}\right)$ at $70 \% \mathrm{RH}$. Plants were watered regularly and supplied with modified half-strength Hoagland nutrient solution once a week (Hoagland and Arnon 1938).

\section{Construction of AprI-overexpressing A. thaliana plants.}

DC3000 aprI was isolated and cloned into pENTRD-TOPO vector generating pENTR_D-TOPO-aprI-stop. Similarly, pENTR_D-TOPO-aprI-fusion was generated, allowing inframe fusion of aprI to GFP. Both constructs with the native SP of AprI and constructs with A. thaliana PR-1 SP (SP PR-1; GenBank At2G14610) replacing the native SP were made. The SP PR-1 was amplified by PCR from $A$. thaliana Col-0 and fused in frame with the mature AprI protein-coding region in plasmids pENTR_D-TOPO-AprI-stop and pENTR_D-TOPOAprI-fusion. Adapted aprI sequences were transferred to pFAST-R02 and pFAST-R05 vectors (Shimada et al. 2010). Plasmids were transferred to Agrobacterium tumefaciens using electroporation. A. thaliana Col-0 plants were transformed with Agrobacterium tumefaciens EHA105 (with pJIC.SaRep plasmid) using the floral dip method (Clough and Bent 1998). Transformed seed were selected based on red fluorescent protein expression using a fluorescence stereomicroscope (MZ16FA; Leica, Wetzlar, Germany).

\section{Bacterial infections.}

P. syringae pv. tomato DC3000 strains were grown for $24 \mathrm{~h}$ in liquid KB medium (King et al. 1954), supplemented with the appropriate antibiotics. Bacteria were collected and resuspended in $10 \mathrm{mM} \mathrm{MgSO}_{4}$ to the proper $\mathrm{OD}_{600}$. For DC3000 infiltration assays in A. thaliana $\left(\mathrm{OD}_{600}=0.0005\right)$, leaves of 4-week-old plants were pressure infiltrated using a syringe without a needle (Van Wees et al. 2013). Plants were placed at $100 \%$ RH. Leaf discs were taken within $2 \mathrm{~h}$ after inoculation $(t=0 ; n=4)$ and 2 days after inoculation $(t=2 ; n=8)$. Subsequently, the leaf discs were ground and $30-\mu l$ aliquots of dilutions were plated onto $\mathrm{KB}$ plates containing rifampicin at $50 \mu \mathrm{g} \mathrm{ml}^{-1}$. After 40 to $48 \mathrm{~h}$ of incubation at $28^{\circ} \mathrm{C}$, bacterial colonies were counted. Infiltration assays with tomato plants were performed as described for A. thaliana, with some modifications. Opposing leaflets of the same leaf of 5-week-old plants were infiltrated with different DC3000 strains and, per plant, two leaves (four leaflets) were infiltrated. Leaf discs were collected within $2 \mathrm{~h}$ after inoculation $(t=0 ; n=3), 4$ days after inoculation $(t=4 ; n$ $=3)$, and 7 days after inoculation $(t=7 ; n=3)$. For gene expression analysis, plants were dip inoculated with a bacterial suspension $\left(\mathrm{OD}_{600}=0.025\right)$ containing $0.015 \%$ (vol/vol) Silwet L-77 (Van Meeuwen Chemicals, Weesp, The Netherlands). Leaf material was harvested directly after inoculation $(t=0 ; n=3)$ and $24 \mathrm{~h}$ after inoculation $(t=24 ; n=3)$.

\section{Gene expression analysis.}

Total RNA from leaf material was isolated as described by Van Wees and associates (1999). Fermentas RevertAid H minus reverse transcriptase (Fermentas, St. Leon-Rot, Germany) was used for cDNA synthesis according to the manufacturer's instructions. Quantitative real-time PCR of the defense-related genes FRK1 (At2G19190) and PR-1 (At2G14610) was performed as described by Verhage and associates (2011). The following primers were used: FRK1 forward $5^{\prime}$-TTT CAA CAG TTG TCG CTG GA-3', FRK1 reverse 5'-AGC TTG CAA TAG CAG GTT GG-3', $P R$ - 1 forward $5^{\prime}$-CTC GGA GCT ACG CAG AAC AAC T-3', $P R-1$ reverse 5'-TTC TCG CTA ACC CAC ATG TTC A-3', PP2AA3 (AT1G13320) forward 5'-TAA CGT GGC CAA AAT GAT GC-3', and $P P 2 A A 3$ reverse 5'-GTT CTC CAC AAC CGC TTG GT-3'. The expression of PP2AA3 (At1G13320) was used for normalization (Czechowski et al. 2005; Hong et al. 2010).

\section{ACKNOWLEDGMENTS}

We thank J. Tommassen and M. Koster for kindly providing pUC4K, pKNG101, pBBR1MCS-5, and E. coli S17-1 $\lambda$ pir; L. Hernández Garcia for pUC4KIXX; and R. Alderliesten and F. Faz Saura for practical assistance. This research was supported by the Centre for BioSystems Genomics and ERC Advanced Investigator Grant number 269072 of the European Research Council.

\section{LITERATURE CITED}

Altschul, S. F., Gish, W., Miller, W., Myers, E. W., and Lipman, D. J. 1990. Basic local alignment search tool. J. Mol. Biol. 215:403-410.

Asai, T., Tena, G., Plotnikova, J., Willmann, M. R., Chiu, W. L., GómezGómez, L., Boller, T., Ausubel, F. M., and Sheen, J. 2002. MAP kinase signalling cascade in Arabidopsis innate immunity. Nature 415:977-983.

Bardoel, B. W., and Van Strijp, J. A. G. 2011. Molecular battle between host and bacterium: Recognition in innate immunity. J. Mol. Recognit. 24:1077-1086.

Bardoel, B. W., Van der Ent, S., Pel, M. J. C., Tommassen, J., Pieterse, C. M. J., Van Kessel, K. P. M., and Van Strijp, J. A. G. 2011. Pseudomonas evades immune recognition of flagellin in both mammals and plants. PLoS Pathog. 7:e1002206.

Bardoel, B. W., Van Kessel, K. P. M., Van Strijp, J. A. G., and Milder, F. J. 2012. Inhibition of Pseudomonas aeruginosa virulence: Characterization of the AprA-AprI interface and species selectivity. J. Mol. Biol. 415:573-583.

Bethesda Research Laboratories. 1986. BRL pUC host: E. coli competent cells. Focus 8:9.

Boller, T., and Felix, G. 2009. A renaissance of elicitors: Perception of microbe-associated molecular patterns and danger signals by patternrecognition receptors. Annu. Rev. Plant Biol. 60:379-406.

Boller, T., and He, S. Y. 2009. Innate immunity in plants: An arms race between pattern recognition receptors in plants and effectors in microbial pathogens. Science 324:742-744.

Bolton, M. D., Van Esse, H. P., Vossen, J. H., De Jonge, R., Stergiopoulos, I., Stulemeijer, I. J. E., Van den Berg, G. C. M., Borras-Hidalgo, O., Dekker, H. L., De Koster, C. G., de Wit, P. J., Joosten, M. H., and Thomma, B. P. 2008. The novel Cladosporium fulvum lysin motif effector Ecp6 is a virulence factor with orthologues in other fungal species. Mol. Microbiol. 69:119-136.

Boyer, H. M., and Roulland-Dussoix, D. 1969. A complementation analysis of the restriction and modification of DNA in Escherichia coli. J. Mol. Biol. 41:459-472.

Buell, C. R., Joardar, V., Lindeberg, M., Selengut, J., Paulsen, I. T., Gwinn, M. L., Dodson, R. J., Deboy, R. T., Durkin, A. S., Kolonay, J. F., Madupu, R., Daugherty, S., Brinkac, L., Beanan, M. J., Haft, D. H., Nelson, W. C., Davidsen, T., Zafar, N., Zhou, L., Liu, J., Yuan, Q. Khouri, H., Fedorova, N., Tran, B., Russell, D., Berry, K., Utterback, T., Van Aken, S. E., Feldblyum, T. V., D’Ascenzo, M., Deng, W.-L., Ramos, A. R., Alfano, J. R., Cartinhour, S., Chatterjee, A. K., Delaney, T. P., Lazarowitz, S. G., Martin, G. B., Schneider, D. J., Tang, X., Bender, C. L., White, O., Fraser, C. M., and Collmer, A. 2003. The complete genome sequence of the Arabidopsis and tomato pathogen Pseudomonas syringae pv. tomato DC3000. Proc. Natl. Acad. Sci. U.S.A. 100:10181-10186.

Chevance, F. F. V., and Hughes, K. T. 2008. Coordinating assembly of a bacterial macromolecular machine. Nat. Rev. Microbiol. 6:455-465.

Clough, S. J., and Bent, A. F. 1998. Floral dip: A simplified method for Agrobacterium-mediated transformation of Arabidopsis thaliana. Plant J. 16:735-743.

Czechowski, T., Stitt, M., Altmann, T., Udvardi, M. K., and Scheible, W. R. 2005. Genome-wide identification and testing of superior reference genes for transcript normalization in Arabidopsis. Plant Physiol. 139:5-17.

De Jonge, R., Van Esse, H. P., Kombrink, A., Shinya, T., Desaki, Y., Bours, R., Van der Krol, S., Shibuya, N., Joosten, M. H. A. J., and Thomma, B. P. H. J. 2010. Conserved fungal LysM effector Ecp6 prevents chitintriggered immunity in plants. Science 329:953-955. 
Duong, F., Lazdunski, A., Cami, B., and Murgier, M. 1992. Sequence of a cluster of genes controlling synthesis and secretion of alkaline protease in Pseudomonas aeruginosa: Relationships to other secretory pathways. Gene 121:47-54.

Felix, G., Duran, J. D., Volko, S., and Boller, T. 1999. Plants have a sensitive perception system for the most conserved domain of bacterial flagellin. Plant J. 18:265-276.

Figurski, D. H., and Helsinki, D. R. 1979. Replication of an origin-containing derivative of plasmid RK2 dependent on a plasmid function provided in trans. Proc. Natl. Acad. Sci. U.S.A. 76:1648-1652.

Galibert, F., Finan, T. M., Long, S. R., Pühler, A., Abola, P., Ampe, F., Barloy-Hubler, F., Barnett, M. J., Becker, A., and Boistard, P. 2001. The composite genome of the legume symbiont Sinorhizobium meliloti. Science 293:668-672.

Gomez-Gomez, L., and Boller, T. 2000. FLS2: An LRR receptor-like kinase involved in the perception of the bacterial elicitor flagellin in Arabidopsis. Mol. Cell 5:1003-1012.

Gomez-Gomez, L., and Boller, T. 2002. Flagellin perception: A paradigm for innate immunity. Trends Plant Sci. 7:251-256.

Guzzo, J., Duong, F., Wandersman, C., Murgier, M., and Lazdunski, A 1991. The secretion genes of Pseudomonas aeruginosa alkaline protease are functionally related to those of Erwinia chrysanthemi proteases and Escherichia coli alpha-haemolysin. Mol. Microbiol. 5:447-453.

Hayashi, F., Smith, K. D., Ozinsky, A., Hawn, T. R., Yi, E. C., Goodlett, D. R., Eng, J. K., Akira, S., Underhill, D. M., and Aderem, A. 2001. The innate immune response to bacterial flagellin is mediated by toll-like receptor 5. Nature 410:1099-1103.

Herrero, M., De Lorenzo, V., and Timmis, K. N. 1990. Transposon vectors containing non-antibiotic resistance selection markers for cloning and stable chromosomal insertion of foreign genes in gram-negative bacteria. J. Bacteriol. 172:6557-6567.

Hoagland, D. R., and Arnon, D. I. 1938. The water culture method for growing plants without soil. Calif. Agric. Exp. Stn. Bull. 347:36-39.

Hong, S. M., Bahn, S. C., Lyu, A., Jung, H. S., and Ahn, J. H. 2010. Identification and testing of superior reference genes for a starting pool of transcript normalization in Arabidopsis. Plant Cell Physiol. 51:1694-1706.

Jones, J. D. G., and Dangl, J. L. 2006. The plant immune system. Nature 444:323-329

Kaniga, K., Delor, I., and Cornelis, G. R. 1991. A wide-host-range suicide vector for improving reverse genetics in gram-negative bacteria: Inactivation of the blaA gene of Yersinia enterocolitica. Gene 109:137-141.

Kerkhoven, R., Van Enckevort, F. H. J., Boekhorst, J., Molenaar, D., and Siezen, R. J. 2004. Visualization for genomics: The microbial genome viewer. Bioinformatics 20:1812-1814.

King, E. O., Ward, M. K., and Raney, D. E. 1954. Two simple media for the demonstration of phycocyanin and fluorescin. J. Lab. Clin. Med. 44:301-307.

Klimpel, K. R., Arora, N., and Leppla, S. H. 1994. Anthrax toxin lethal factor contains a zinc metalloprotease consensus sequence which is required for lethal toxin activity. Mol. Microbiol. 13:1093-1100.

Komoriya, K., Shibano, N., Higano, T., Azuma, N., Yamaguchi, S., and Aizawa, S. 1999. Flagellar proteins and type III-exported virulence factors are the predominant proteins secreted into the culture media of Salmonella typhimurium. Mol. Microbiol. 34:767-779.

Kovach, M. E., Phillips, R. W., Elzer, P. H., Roop, R. M., and Peterson, K M. 1994. pBBR1MCS: A broad-host-range cloning vector. Biotechniques 16:800-802.

Kunze, G., Zipfel, C., Robatzek, S., Niehaus, K., Boller, T., and Felix, G. 2004. The $\mathrm{N}$ terminus of bacterial elongation factor tu elicits innate immunity in Arabidopsis plants. Plant Cell 16:3496-3507.

Létoffé, S., Delepelaire, P., and Wandersman, C. 1989. Characterization of a protein inhibitor of extracellular proteases produced by Erwinia chrysanthemi. Mol. Microbiol. 3:79-86.

Létoffé, S., Delepelaire, P., and Wandersman, C. 1990. Protease secretion by Erwinia chrysanthemi: The specific secretion functions are analogous to those of Escherichia coli alpha-hemolysin. EMBO (Eur. Mol. Biol. Organ.) J. 9:1375-1382.

Liehl, P., Blight, M., Vodovar, N., Boccard, F., and Lemaitre, B. 2006. Prevalence of local immune response against oral infection in a Drosophila/Pseudomonas infection model. PLoS Pathog. 2:551-561.

Mansfield, J., Genin, S., Magori, S., Citovsky, V., Sriariyanum, M. Ronald, P., Dow, M., Verdier, V., Beer, S. V., Machado, M. A., Toth, I., Salmond, G., And Foster, G. D. 2012. Top 10 plant pathogenic bacteria in molecular plant pathology. Mol. Plant Pathol. 13:614-629.

Miyoshi, S., and Shinoda, S. 2000. Microbial metalloproteases and pathogenesis. Microb. Infect. 2:91-98.

Parmely, M., Gale, A., Clabaugh, M., Horvat, R., and Zhou, W. W. 1990. Proteolytic inactivation of cytokines by Pseudomonas aeruginosa. Infect. Immun. 58:3009-3014.
Pel, M. J. C., and Pieterse, C. M. J. 2013. Microbial recognition and evasion of host immunity. J. Exp. Bot. 64:1237-1248.

Preston, G. M. 2000. Pseudomonas syringae pv. tomato: The right pathogen, of the right plant, at the right time. Mol. Plant Pathol. 1:263-275.

Ramos, H. C., Rumbo, M., and Sirard, J. C. 2004. Bacterial flagellins: Mediators of pathogenicity and host immune responses in mucosa. Trends Microbiol. 12:509-517.

Ronald, P. C., and Beutler, B. 2010. Plant and animal sensors of conserved microbial signatures. Science 330:1061-1064.

Samatey, F. A., Imada, K., Nagashima, S., Vonderviszt, F., Kumasaka, T., Yamamoto, M., and Namba, K. 2001. Structure of the bacterial flagellar protofilament and implications for a switch for supercoiling. Nature 410:331-337.

Shan, L. B., He, P., Li, J. M., Heese, A., Peck, S. C., Nürnberger, T., Martin, G. B., and Sheen, J. 2008. Bacterial effectors target the common signaling partner BAK1 to disrupt multiple MAMP receptor-signaling complexes and impede plant immunity. Cell Host Microbe 4:17-27.

Shimada, T. L., Shimada, T., and Hara-Nishimura, I. 2010. A rapid and non-destructive screenable marker, FAST, for identifying transformed seeds of Arabidopsis thaliana. Plant J. 61:519-528.

Silby, M. W., Cerdeno-Tarraga, A. M., Vernikos, G. S., Giddens, S. R., Jackson, R. W., Preston, G. M., Zhang, X. X., Moon, C. D., Gehrig, S. M., Godfrey, S. A. C., Knight, C. G., Malone, J. G., Robinson, Z., Spiers, A. J., Harris, S., Challis, G. L., Yaxley, A. M., Harris, D., Seeger, K., Murphy, L., Rutter, S., Squares, R., Quail, M. A., Saunders, E., Mavromatis, K., Brettin, T. S., Bentley, S. D., Hothersall, J., Stephens, E., Thomas, C. M., Parkhill, J., Levy, S. B., Rainey, P. B., and Thomson, N. R. 2009. Genomic and genetic analyses of diversity and plant interactions of Pseudomonas fluorescens. Genome Biol. 10:R51.

Smith, K. D., Andersen-Nissen, E., Hayashi, F., Strobe, K., Bergman, M. A., Barrett, S. L. R., Cookson, B. T., and Aderem, A. 2003. Toll-like receptor 5 recognizes a conserved site on flagellin required for protofilament formation and bacterial motility. Nat. Immunol. 4:1247 1253

Stover, C., Pham, X., Erwin, A., Mizoguchi, S., Warrener, P., Hickey, M., Brinkman, F., Hufnagle, W., Kowalik, D., Lagrou, M., Garber, R. L., Goltry, L., Tolentino, E., Westbrock-Wadman, S., Yuan, Y., Brody, L. L., Coulter, S. N., Folger, K. R., Kas, A., Larbig, K., Lim, R., Smith, K. Spencer, D., Wong, G. K.-S., Wu, Z., Paulsen, I. T., Reizer, J., Saier, M. H., Hancock, R. E. W., Lory, S., and Olson, M. V. 2000. Complete genome sequence of Pseudomonas aeruginosa PAO1, an opportunistic pathogen. Nature 406:959-964.

Taylor, L. A., and Rose, R. E. 1988. A correction in the nucleotidesequence of the Tn903 kanamycin resistance determinant in Puc4k Nucleic Acids Res. 16:358-358.

Tommassen, J., Filloux, A., Bally, M., Murgier, M., and Lazdunski, A. 1992. Protein secretion in Pseudomonas aeruginosa. FEMS (Fed. Eur. Microbiol. Soc.) Microbiol. Rev. 9:73-90.

Van Wees, S. C. M., Luijendijk, M., Smoorenburg, I., Van Loon, L. C., and Pieterse, C. M. J. 1999. Rhizobacteria-mediated induced systemic resistance (ISR) in Arabidopsis is not associated with a direct effect on expression of known defense-related genes but stimulates the expression of the jasmonate-inducible gene Atvsp upon challenge. Plant Mol. Biol. 41:537-549.

Van Wees, S. C. M., Van Pelt, J. A., Bakker, P. A. H. M., and Pieterse, C. M. J. 2013. Bioassays for assessing jasmonate-dependent defenses triggered by pathogens, herbivorous insects, or beneficial rhizobacteria. Methods Mol. Biol. 1011:35-49.

Verhage, A., Vlaardingerbroek, I., Raaymakers, C., Van Dam, N., Dicke, M., Van Wees, S. C. M., and Pieterse, C. M. J. 2011. Rewiring of the jasmonate signaling pathway in Arabidopsis during insect herbivory. Front. Plant Sci. 2:1-12.

Vieira, J., and Messing, J. 1982. The pUC plasmids, an M13mp7-derived system for insertion mutagenesis and sequencing with synthetic universal primers. Gene 19:259-268.

Whalen, M. C., Innes, R. W., Bent, A. F., and Staskawicz, B. J. 1991. Identification of Pseudomonas syringae pathogens of Arabidopsis and a bacterial locus determining avirulence on both Arabidopsis and soybean. Plant Cell 3:49-59.

Wyant, T. L., Tanner, M. K., and Sztein, M. B. 1999. Salmonella typhi flagella are potent inducers of proinflammatory cytokine secretion by human monocytes. Infect. Immun. 67:3619-3624.

Zeng, W., and He, S.-Y. 2010. A prominent role of the flagellin receptor FLAGELLIN-SENSING2 in mediating stomatal response to Pseudomonas syringae pv. tomato DC3000 in Arabidopsis. Plant Physiol. 153:1188-1198

Zipfel, C., Robatzek, S., Navarro, L., Oakeley, E. J., Jones, J. D. G., Felix, G., and Boller, T. 2004. Bacterial disease resistance in Arabidopsis through flagellin perception. Nature 428:764-767. 